\title{
Efeito dose-resposta de uma formulação de dentifrício com concentração reduzida de fluoreto - estudo in vitro
}

\section{Dose-response effect of a dentifrice formulation with low fluoride concentration - an in vitro study}

\author{
Helena Maria Decico Negri* \\ Jaime Aparecido Cury**
}

\begin{abstract}
RESUMO: O desenvolvimento de dentifrício com menor concentração de flúor, visando crianças menores de 6 anos, tem sido sugerido para diminuir o risco de fluorose dental. Para que um dentifrício tenha potencial anticárie é necessário que ele forme no esmalte dental flúor fracamente ligado tipo fluoreto de cálcio $\left(\mathrm{CaF}_{2}\right)$, o qual é considerado o responsável pelo efeito do flúor tópico. Considerando que a formação de $\mathrm{CaF}_{2}$ é função inversa do $\mathrm{pH}$, foram formulados dentifrícios com pH 5,5. Foram preparados dentifrícios com 275, 550 e 1.100 ppm F' ${ }^{-}$contendo NaF/sílica, para verificar efeito dose-resposta os quais foram comparados com um placebo e o dentifrício Crest ${ }^{\circledR}$ (controle positivo - padrão). A biodisponibilidade dos dentifrícios, em termos de formação de flúor total (FT), $\mathrm{CaF}_{2}$ e fluorapatita (FA) no esmalte dental humano foi avaliada. Para as dosagens de íon flúor foi utilizado eletrodo específico. Os resultados mostraram que a formulação com 550 ppm de $\mathrm{F}^{-}$foi mais eficiente que o placebo e aquela com $275 \mathrm{ppm}$ em termos de formação de $\mathrm{CaF}_{2}(\mathrm{p}<0,05)$. Em acréscimo, ela não diferiu do dentifrício padrão ( $\left.\mathrm{p}>0,05\right)$. Uma relação dose-efeito foi observada em termos de $\mathrm{CaF}_{2}$ formado. Os resultados sugerem que a formulação modificada contendo 550 ppm de $\mathrm{F}^{-}$pode ser considerada equivalente ao dentifrício padrão com 1.100 ppm, a qual seria mais segura em termos de fluorose dental.

DESCRITORES: Dentifrícios; Flúor; Cárie; Fluorose dentária.
\end{abstract}

\begin{abstract}
The utilization of dentifrices with low fluorine concentration, for children under 6 years of age, has been suggested to reduce the risks of dental fluorosis. However, in order to have anticariogenic potential, the dentifrice should form loosely-bound fluorine $\left(\mathrm{CaF}_{2}\right)$ on dental enamel. Considering that the formation of $\mathrm{CaF}_{2}$ is a function inversely related to $\mathrm{pH}$, dentifrices with $\mathrm{pH} 5.5$, with 275,550 and $1,100 \mathrm{ppm} \mathrm{F}^{-}$(NaF/silica) were developed in order to assess dose-response effects. A comparison between those dentifrices, a placebo product and the Crest toothpaste (positive control - standard) was carried out. Furthermore, the bioavailability of dentifrices, in terms of formation of total fluorine (TF), $\mathrm{CaF}_{2}$, and fluorapatite (FA) on human dental enamel, was evaluated. An ion-specific electrode was utilized for the determination of the dosage of fluorine. The results revealed that the dentifrice with $550 \mathrm{ppm} \mathrm{F}^{-}$was more effective than both the placebo and the dentifrice with $275 \mathrm{ppm}$, presenting no difference in relation to the positive control ( $p$ > 0.05). A dose-effect correlation was observed as to the $\mathrm{CaF}_{2}$ formed. In conclusion, the modified formulation with $550 \mathrm{ppm} \mathrm{F}^{-}$can be considered as effective as the standard dentifrice with $1,100 \mathrm{ppm}$, and its utilization would be safer with regard to dental fluorosis.
\end{abstract}

DESCRIPTORS: Dentifrices; Fluorine; Caries; Fluorosis, dental.

\section{INTRODUÇÃO}

A partir da década de 80 , um declínio de cárie começou a ser relatado em países desenvolvidos (Hargreaves et al. ${ }^{9}, 1990 ;$ Newbrun $^{16}$, 1992). Na busca por razões para tal fato, chegou-se ao consenso de que o único fator comum que poderia explicar essa constatação era uma coincidência entre o aumento da oferta de dentifrícios fluoretados e a conseqüente redução de cárie (Rölla et $a l^{22}$, 1991). Um declínio de cárie dental também passou a ser notado em países em desenvolvimento dez anos após, como o Brasil (Narvai et al. ${ }^{15}$, 1999) e tem sido sugerido que dentifrícios fluoretados tiveram papel relevante neste fenômeno $\left(\mathrm{Cury}^{4}, 2001\right)$.

Por outro lado, notou-se um aumento na prevalência de fluorose dental, em países desenvolvidos em regiões contendo ou não água fluoretada, justificada pelo fato de que crianças em idade pré-escolar não possuem total controle sobre os músculos da deglutição, ingerindo em media 50\% do dentifrício durante a escovação (Lima, Cury ${ }^{12}, 2001$; Paiva, Cury ${ }^{17}$, 2001). Considerando então os dentifrícios fluoretados como um fator de risco de fluorose dental (Hargreaves et al. ${ }^{9}, 1970$; Dowel ${ }^{5}$, 1981; Mascarenhas ${ }^{13}$ 2000) seria desejável por parte dos

\footnotetext{
* Mestre em Odontopediatria pela Faculdade de Odontologia de Araraquara.

** Professor Titular de Bioquímica da Faculdade de Odontologia de Piracicaba da Universidade Estadual de Campinas.
} 
Negri HMD, Cury JA. Efeito dose-resposta de uma formulação de dentifrício com concentração reduzida de fluoreto - estudo in vitro. Pesqui Odontol Bras 2002;16(4):361-365.

fabricantes uma redução na concentração de fluoreto $\left(\mathrm{F}^{-}\right)$nos dentifrícios destinados a população infantil.

Entretanto para que um dentifrício fluoretado seja eficiente, alguns requisitos mínimos são necessários como: presença de fluoreto solúvel, estabilidade, biodisponibilidade do fluoreto e a sua capacidade em inibir a desmineralização e ativar a remineralização do esmalte $\left(\mathrm{ADA}^{1}\right.$, 1985). Atualmente há um consenso de que o fluoreto fracamente ligado tipo fluoreto de cálcio $\left(\mathrm{CaF}_{2}\right)$ é o responsável pelo efeito anticárie do flúor tópico (Saxegaard, Rölla ${ }^{24}$, 1989; Larsen $^{11}$, 2001). Sabe-se também que a formação deste produto no esmalte é dependente do $\mathrm{pH}$. Desta maneira, reduzindo-se o $\mathrm{pH}$ do dentifrício pode-se aumentar a quantidade $\mathrm{de} \mathrm{CaF}_{2}$ formada no esmalte, assim poder-se-ia ter um dentifrício com menor concentração de fluoreto e o mesmo efeito anticárie que um dentifrício convencional com 1.000-1.100 ppm F.

Assim, Petersson et al. ${ }^{19}$ (1989) demonstraram in vitro que um dentifrício com $\mathrm{pH} 5,5$ e com 250 ppm de $\mathrm{F}^{-}$forma aproximadamente a mesma quantidade de fluoreto na superfície do esmalte que um dentifrício com pH 7,0 e 1.100 ppm FEntretanto este estudo não foi realizado em comparação com o dentifrício padrão ("Gold Standard”), não sendo também avaliada a relação dose-efeito que tem sido uma recomendação atual (Proskin $^{20}$, 1995). Em acréscimo, a metodologia de análise utilizada foi qualitativa e não era capaz de diferenciar a formação de $\mathrm{CaF}_{2}$.

Portanto este trabalho tem como objetivo avaliar in vitro uma formulação modificada de dentifrício em termos de relação dose-efeito e em relação ao dentifrício padrão, avaliando sua reatividade com o esmalte dental em condições que simulam o ato de escovação.

\section{MATERIAL E MÉTODO Dentifrícios utilizados}

As formulações avaliadas nesta pesquisa foram preparadas pela Kolynos do Brasil - São Bernardo do Campo, seguindo orientações do Laboratório de Bioquímica Oral da FOP-UNICAMP. Assim foram formulados cinco dentifrícios à base de sílica e com $\mathrm{pH}$ 5,5. Assim, foram constituídos os seguintes grupos experimentais:

- formulação sem agregação de sais de flúor controle negativo;
- formulações experimentais contendo fluoreto de sódio a 275 ppm, 550 ppm e 1.100 ppm de $\mathrm{F}$;

- dentifrício Crest ${ }^{\circledR}$ contendo 1.100 ppm F ${ }^{-}$- controle positivo.

Todos os dentifrícios foram codificados para que o estudo fosse do tipo cego.

\section{Reatividade com o esmalte dental Preparo de particulas de esmalte}

Para determinação da reatividade dos dentifrícios com o esmalte dental, foram utilizados coroas de terceiros molares inclusos armazenados em formol $2 \%$ e pH 7,0. As coroas foram imersas em clorofórmio para retirada de gordura e passaram por lavagem com metanol e levadas à estufa para secagem. Para pulverização do esmalte foi utilizado moinho de bolas (Microyeur Quantitafif Dangoumau, Prolabo, Paris) e em seguida com auxílio de tamis (Telatest) as partículas de diâmetro de 0,075 a $0,149 \mathrm{~mm}$ foram selecionadas. A dentina foi separada do esmalte utilizando solução bromofórmio e acetona (92:8), após o que, o pó de esmalte foi lavado em água deionizada e levado à estufa para secagem. O uso de partículas de esmalte permite aumentar a área de reação e seu uso é aceito na literatura mundial para avaliar a reatividade do fluoreto (Melo Franco, Cury ${ }^{14}$, 1994).

\section{Preparo da solução de dentifricio e teste de reatividade}

A capacidade dos dentifrícios reagirem com o esmalte dental foi determinada através das concentrações de fluoreto total, fluorapatita e fluoreto de cálcio formados no esmalte. Inicialmente dilui-se o dentifrício em água destilada e desmineralizada na proporção de $1: 3$, que é a diluição que o dentifrício sofre no meio bucal (Duke, Forward 6 , 1982). A seguir as partículas de esmalte, 20,0 mg, foram pré-tratadas com saliva humana estimulada (Tabchoury, Cury ${ }^{25}, 1994$ ), após o que, a saliva foi desprezada e as partículas de esmalte foram tratadas durante 1 minuto com $20,0 \mathrm{ml}$ de sobrenadante da suspensão de dentifrício. A seguir esperou-se 1 minuto para que ocorresse a decantação quando finalmente as partículas de esmalte foram filtradas à vácuo, lavadas com água, metanol e secas por 1 hora à $90^{\circ} \mathrm{C}$, sendo neste esmalte determinadas as concentrações de fluoreto total, fluoreto de cálcio e fluorapatita, de acordo com procedimentos descrito por Cury $^{3}$ (1987). Foram realizadas seis repetições para cada análise. 
Negri HMD, Cury JA. Efeito dose-resposta de uma formulação de dentifrício com concentração reduzida de fluoreto - estudo in vitro. Pesqui Odontol Bras 2002;16(4):361-365.

\section{Determinação de fluoreto total (FT)}

Inicialmente 5,0 mg de esmalte foram pesados em balança analítica (Ohaus, $\pm 0,0001$ ). Após a desmineralização do esmalte por 1 hora com $0,5 \mathrm{ml}$ de $\mathrm{HCl}$ 1,0 M sob agitação foi acrescentado ao ácido $2,0 \mathrm{ml}$ de citrato trissódico a 0,5 M. As concentrações em ppm $\mathrm{F}^{-}$foram calculadas de acordo com a quantidade de pó de esmalte presente na amostra, após a leitura com eletrodo específico.

\section{Determinação de fluoreto de cálcio $\left(\mathrm{CaF}_{2}\right)$}

Foi pesado em balança analítica 10,0 mg de pó de esmalte os quais foram transferidos para tubo de centrífuga plástico. Ao tubo foi adicionado 1,0 ml de KOH 1,0 M, após 24 horas sob agitação extraiu-se o fluoreto alcali solúvel (Caslavska et $\left.a .^{2}, 1975\right)$. Após centrifugação por 5 minutos a $3.500 \mathrm{rpm}$ (centrífuga Excelsa Baby II), 0,5 ml do sobrenadante foi transferido para tubo plástico. Ao tubo foi adicionado $1,0 \mathrm{ml}$ de citrato trissódico 1,0 M e 1,0 ml de $\mathrm{HCl}$ 1,0 M. As concentrações de íon flúor foram obtidas à semelhança com flúor total (FT).

\section{Determinação da fluorapatita (FA)}

Após a extração por 24 horas do fluoreto alcali solúvel $\left(\mathrm{CaF}_{2}\right)$, o precipitado foi lavado com água deionizada por 3 vezes e 1 vez com metanol. Uma quantidade de 5,0 mg do pó de esmalte seco foi pesada e desmineralizada com $0,5 \mathrm{ml}$ de $\mathrm{HCl} 1,0 \mathrm{M}$ por 1 hora sob agitação, após o que foi adicionado $2,0 \mathrm{ml}$ de citrato trissódico $0,5 \mathrm{M}$. As concentrações de flúor foram determinadas de modo semelhante ao FT.

\section{Análise de fluoreto}

Para determinação de fluoreto nas formas de FT, $\mathrm{CaF}_{2}$ e FA utilizou-se eletrodo específico para íons flúor Orion 96-09 e analisador de íons Orion EA 940, previamente às leituras uma curva de calibração foi testada com soluções padrões de íons flúor preparadas com citrato trissódico $0,4 \mathrm{M} \mathrm{e} \mathrm{HCl}$ $0,2 \mathrm{M}$ contendo de 0,08 a $0,8 \mu \mathrm{g} \mathrm{\textrm {F } ^ { - }} / \mathrm{ml}$. As leituras de todas as amostras e padrões foram realizadas sob agitação (agitador Marte e barras magnéticas). Tanto as calibrações como os cálculos foram feitos com o auxílio do software Quattro-Pro.

\section{Análise estatística}

As variáveis concentrações de fluoreto total (FT), fluorapatita (FA) e fluoreto de cálcio $\left(\mathrm{CaF}_{2}\right)$ foram analisadas com seis repetições. $\mathrm{O}$ único fator avaliado foi o dentifrício. A análise mais apropriada foi a análise de variância não-paramétrica para o delineamento inteiramente ao acaso. O teste não-paramétrico utilizado foi o de Kruskal-Wallis, e as médias foram comparadas através de comparações múltiplas também não-paramétricas, com nível de significância de 5\%. A relação dose-efeito foi avaliada por regressão polinominal quadrática.

\section{RESULTADOS}

Na Tabela 1 são mostradas as médias e desvios padrões das médias, das concentrações de fluoreto (ppm F-) no esmalte em função dos tratamentos.

Para o fluoreto total (FT), a maior média foi observada com a formulação contendo 1.100 ppm F que diferiu significativamente dos demais, sendo a menor média encontrada com o tratamento placebo que por sua vez também diferiu significativamente dos demais.

Para a concentração de fluorapatita (FA) a maior média foi observada com a formulação 1.100 ppm $\mathrm{F}^{-}$, que não diferiu do dentifrício Crest ${ }^{\circledR}$ e do dentifrício $550 \mathrm{ppm} \mathrm{F}^{-}$, esse por sua vez diferiu do dentifrício $275 \mathrm{ppm} \mathrm{F}$; todos esses diferiram significativamente do dentifrício placebo.

Para a concentração de fluoreto de cálcio $\left(\mathrm{CaF}_{2}\right)$ formado no esmalte dental, a maior média foi observada com a formulação 1.100 ppm $\mathrm{F}^{-}$que diferiu significativamente dos demais. A formulação com 550 ppm e o dentifrício comercial Crest ${ }^{\circledR}$ não diferiram entre si e diferiram dos demais. A menor concentração foi observada com o dentifrício placebo que diferiu de todos os outros tratamentos, seguido do tratamento 275 ppm $\mathrm{F}^{-}$, o qual também diferiu significativamente dos demais.

TABELA 1 - Médias e desvios padrão da média $(n=6)$ da concentração de fluoreto no esmalte (ppm F-) de acordo com os tratamentos.

\begin{tabular}{l|c|c|c}
\hline \hline \multirow{2}{*}{ Dentifrícios } & \multicolumn{3}{|c}{ ppm F no esmalte dental } \\
\cline { 2 - 4 } & FT & FA & CaF $_{2}$ \\
\hline Placebo & $71,4 \pm 1,7 \mathrm{~d}$ & $67,7 \pm 1,7 \mathrm{c}$ & $79,7 \pm 1,3 \mathrm{~d}$ \\
\hline $275 \mathrm{ppm}$ & $180,4 \pm 5,2 \mathrm{c}$ & $97,4 \pm 4,4 \mathrm{~b}$ & $165,1 \pm 7,9 \mathrm{c}$ \\
\hline $550 \mathrm{ppm}$ & $231,1 \pm 12,8 \mathrm{~b}$ & $109,1 \pm 3,4 \mathrm{ba}$ & $224,4 \pm 8,9 \mathrm{~b}$ \\
\hline $1.100 \mathrm{ppm}$ & $287,9 \pm 9,8 \mathrm{a}$ & $120,2 \pm 2,1 \mathrm{a}$ & $275,1 \pm 5,8 \mathrm{a}$ \\
\hline Crest $^{\circledR}$ & $210,8 \pm 5,7 \mathrm{~b}$ & $111,4 \pm 3,8 \mathrm{a}$ & $205,9 \pm 6,4 \mathrm{~b}$ \\
$(1.100 \mathrm{ppm})$ & & & \\
\hline \hline
\end{tabular}

FT = flúor total; FA = fluorapatita. Tratamentos cujas médias são seguidas por letras distintas diferem estatisticamente a $5 \%$. 
Uma relação dose-efeito estatisticamente significativa foi encontrada para $\mathrm{CaF}_{2}$ formado no esmalte $(p=0,0104)$. Para FT e FA a relação não foi significativa ( $p>0,05)$.

\section{DISCUSSÃO}

Tendo em vista a relação entre aumento da prevalência de fluorose dental e uso de dentifrícios fluoretados, várias medidas têm sido sugeridas para garantir que o declínio de cárie dental alcançado mundialmente continue sem efeitos colaterais indesejáveis. Podemos citar algumas recomendações sugeridas para a escovação dental em crianças menores de seis anos de idade: 1) não usar dentifrícios, ou usar um dentifrício não fluoretado $\left(\mathrm{Feigal}^{8}, 1983\right)$, porém fluoreto tem sido indispensável no controle de cárie quando da escovação dental; 2) usar uma pequena quantidade de dentifrício para escovar os dentes, entretanto a eficiência anticárie deste procedimento não está ainda comprovada (Ericsson, Forsman $\left.^{7}, 1969\right)$; 3) usar um dentifrício com concentração reduzida de fluoreto (Pendrys ${ }^{18}$, 1995), entretanto a eficácia de dentifrício com concentrações abaixo de 1.000 ppm $\mathrm{F}^{-}$não está bem estabelecida (Richards, Banting ${ }^{21}$, 1996).

Entre as alternativas muito se tem discutido sobre a possibilidade de ser reduzida a concentração de flúor dos dentifrícios infantis. Entretanto, simplesmente diminuir a concentração pode aumentar a segurança, mas pode comprometer o benefício de controle do desenvolvimento da cárie. Assim, se o risco de cárie é baixo um dentifrício com metade da concentração de flúor poderia ter o mesmo efeito que o convencional a 1.000-1.100 ppm F'. Por outro lado, o ideal seria desenvolver uma formulação com flúor mais ativo o que permitiria aumentar a segurança sem risco de perda de efeito, independente da atividade de cárie a ser controlada. Deste modo, o objetivo do presente trabalho foi avaliar uma formulação desenvolvida para ter flúor mais reativo de tal forma que $550 \mathrm{ppm} \mathrm{F}^{-}$tivesse o mesmo efeito que um dentifrício convencional a 1.100 ppm F.
Os resultados obtidos com relação a reatividade do fluoreto da formulação desenvolvida mostram que este objetivo foi conseguido (Tabela 1). Uma relação dose-efeito foi observada em termos de $\mathrm{CaF}_{2}$ formado no esmalte dental $(p=0,0104)$. Considerando que $\mathrm{CaF}_{2}$ é o principal produto em termos de efeito de um método tópico (Saxegaard, Rölla ${ }^{24}$, 1989; Ten Cate et $a{ }^{26}{ }^{26}$, 1998), a quantidade formada aumenta em função da concentração de flúor nas formulações de zero a 1.100 ppm. Em acréscimo o dado mais relevante é que a formulação com 550 ppm de $\mathrm{F}^{-}$tem uma capacidade de formar $\mathrm{CaF}_{2}$ no esmalte tanto quanto o dentifrício comercial Crest ${ }^{\circledR}$ que é considerado padrão para equivalência de efeito. $O$ dentifrício Crest $^{\circledast}$ é a base de sílica e contém 1.100 ppm F na forma de NaF. A formulação desenvolvida tem a mesma base, mas sendo mais reativa, com a metade da concentração de flúor forma a mesma quantidade de " $\mathrm{CaF}_{2}$ " no esmalte.

Assim, tendo em vista a maior reatividade do flúor com o esmalte dental, espera-se do dentifrício desenvolvido com 550 ppm $\mathrm{F}^{-}$uma maior capacidade de interferir com o desenvolvimento de cárie.

\section{CONCLUSÕES}

A formulação desenvolvida é mais reativa mostrando dose-efeito em termos de formação de $\mathrm{CaF}_{2}$ no esmalte.

Por ser mais reativa, a formulação com 550 ppm $\mathrm{F}^{-}$é equivalente ao dentifrício padrão $\mathrm{Crest}^{\circledR} \mathrm{em}^{-}$termos de $\mathrm{CaF}_{2}$ formado no esmalte dental.

Os resultados sugerem que a formulação desenvolvida pode ter o mesmo efeito clínico que uma convencional com $1.100 \mathrm{ppm} \mathrm{F}$, porém com a metade da concentração (550 ppm F), a qual seria portanto mais segura em termos de fluorose dental.

\section{AGRADECIMENTOS}

A FAPESP pela concessão da bolsa de mestrado (processo 96/1826-2). Ao Sr. Waldomiro Vieira Filho, Sra. Mariza de Jesus Carlos Soares e Sr. José Alfredo da Silva, técnicos do laboratório de Bioquímica Oral da FOP-UNICAMP, pelo auxílio nas análises.

\section{REFERÊNCIAS}

1. ADA - Council on Dental Therapeutics. Guidelines for the acceptance of fluoride-containing dentifrices. J Am Dent Assoc 1985;110:545-7.

2. Caslavska V, Moreno EC, Brudevold F. Determination of the calcium fluoride formed from in vitro exposure of human enamel to fluoride solutions. Archs Oral Biol 1975;20:333-9.
3. Cury JA. Avaliação de um gel de dentifrício contendo xilitol. Rev Bras Odontol 1987;44:36-40.

4. Cury JA. Uso do flúor e controle da cárie como doença. In: Baratieri LN, Monteiro S, Andrada MAC, Vieira LCC, Ritter AV, Cardoso AC. Odontologia Restauradora: fundamentos e possibilidades. São Paulo: Santos, 2001. p. 33-68. 
Negri HMD, Cury JA. Efeito dose-resposta de uma formulação de dentifrício com concentração reduzida de fluoreto - estudo in vitro. Pesqui Odontol Bras 2002;16(4):361-365.

5. Dowel TD. The use of toothpaste in infancy. Br Dent $\mathrm{J}$ 1981;4:247-9.

6. Duke SA, Forward GC. The conditions occurring in vivo when brushing with toothpaste. Br Dent J 1982;152:52-4.

7. Ericsson Y, Forsman B. Fluoride retained from mouthrinses and dentifrices in preschool children. Caries Res 1969;3:290-9.

8. Feigal RJ. Recent modifications in use of fluoride for children. Northwest Dent 1983;62:19-21.

9. Hargreaves JA, Ingram GS, Wagg BJ. Excretion studies on the ingestion of monofluophosphate toothpaste by children. Caries Res 1970;4:256-68.

10. Hargreaves JA, Cleaton-Jones E. Dental caries changes in the Scottish Isle of Lewis. Caries Res 1990;24:137-41.

11. Larsen MJ, Richards A. The influence of saliva on the formation of calcium fluoride material on human dental enamel. Caries Res 2001;35:57-60.

12. Lima YBO, Cury JA. Ingestão de flúor por crianças através da água e dentifrícios. Rev Saúde Pública 2001;35:576-81.

13. Mascarenhas AK. Risk factors for dental fluorosis: a review of the recent literature. Pediatric Dent 2000;22;269-77.

14. Melo Franco E, Cury JA. Effect of prebrushing rinse Plax on the deposition of fluoride on enamel. Am J Dent 1994;7:119-21.

15. Narvai PC, Frazão P, Castellanos RA. Declínio na experiência de cárie em dentes permanentes de escolares brasileiros no final de século XX. Odontologia e Sociedade 1999;1:25-9.
16. Newbrun E. Current regulations and recommendations concerning water fluoridation, fluoride supplements and topical fluoride agents. J Dent Res 1992;1255-65.

17. Paiva SM, Cury JA. Dentifrício fluoretado e risco de fluorose dentária. RPG Rev Pós Grad 2001;8:322-8.

18. Pendrys DG. Risk of fluorosis in a fluoridated population. J Am Dent Assoc 1995;126:1617-24.

19. Petersson LG, Lodding A, Hakeberg M, Koch G. Fluorine profiles in human after in vitro treatment with dentifrices of different compositions and acidities. Swed Dent J 1989;13:177-83.

20. Proskin HM. Statistical considerations related to the use of caries model systems for determination of the clinical effectiveness of therapeutic agents. Adv Dent Res 1995;9:270-9.

21. Richards A, Banting DW. Fluoride toothpastes. In: Fejerskov O, Ekstrand J, Burt BA. Fluoride in dentistry. $2^{\text {nd }}$ ed. Copenhagen: Munksgaard; 1996. p. 328-43.

22. Rölla G, Ogaard B, Cruz RA. Clinical effect and mechanism of cariostatic action of fluoride-containing toothpastes: a review. Int Dent J 1991;41:171-4.

23. Saxegaard E, Rölla G. Fluoride acquisition on and in human enamel during topical application in vivo. Scand $\mathrm{J}$ Dent Res 1988;96:523-35.

24. Saxegaard E, Rölla G. Kinetics of acquisition and loss of calcium fluoride by enamel in vivo. Caries Res 1989;23:406-11.

25. Tabchoury CPM, Cury JA. Estudo de condições de envelhecimento precoce de dentifrícios para prever o comportamento do flúor em condições ambientais. Rev Bras Farm 1994;75:67-71.

26. Ten Cate JM, Dames JJ, Buijis MJ. Inhibition of demineralization by fluoride in vitro. Caries Res 1998;32;141-7.

Recebido para publicação em 10/01/02 Enviado para reformulação em 22/07/02 Aceito para publicação em 08/08/02 